\title{
Thermally Driven Inhibition of Superconducting Vortex Avalanches
}

\author{
Antonio Lara, ${ }^{1}$ Farkhad G. Aliev, ${ }^{1, *}$ Victor V. Moshchalkov, ${ }^{2}$ and Yuri M. Galperin ${ }^{3,4}$ \\ ${ }^{1}$ Departamento Física de la Materia Condensada, INC and IFIMAC, \\ Universidad Autónoma de Madrid, 28049 Madrid, Spain \\ ${ }^{2}$ INPAC-Katholieke Universiteit Leuven, Celestijnenlaan 200D, B3001 Leuven, Belgium \\ ${ }^{3}$ Department of Physics, University of Oslo, 0316 Oslo, Norway \\ ${ }^{4}$ A. F. Ioffe Physico-Technical Institute of Russian Academy of Sciences, 194021 St. Petersburg, Russia \\ (Received 30 June 2017; revised manuscript received 20 August 2017; published 26 September 2017) \\ Complex systems close to their critical state can exhibit abrupt transitions-avalanches-between their \\ metastable states. It is a challenging task to understand the mechanism of the avalanches and control their \\ behavior. Here, we investigate microwave stimulation of avalanches in the so-called vortex matter of type-II \\ superconductors - a system of interacting Abrikosov vortices close to the critical (Bean) state. Our main \\ finding is that the avalanche incubation strongly depends on the excitation frequency, a completely \\ unexpected behavior observed close to the so-called depinning frequencies. Namely, the triggered vortex \\ avalanches in $\mathrm{Pb}$ superconducting films become effectively inhibited approaching the critical temperature \\ or critical magnetic field when the microwave stimulus is close to the vortex depinning frequency. \\ We suggest a simple model explaining the observed counterintuitive behaviors as a manifestation of the \\ strongly nonlinear dependence of the driven vortex core size on the microwave excitation intensity. This \\ paves the way to controlling avalanches in superconductor-based devices through their nonlinear response.
}

DOI: 10.1103/PhysRevApplied.8.034027

\section{INTRODUCTION}

Control over stability of vortex matter in type-II superconducting films and materials is essential for a broad range of current and future potential applications. Penetration of magnetic flux in the form of vortex avalanches is the most harmful for the real devices ranging from microwave resonators, bolometers, superconducting quantum interference devices, or superconducting tapes (see, e.g., Ref. [1]).

In general, an avalanche is an apparently unpredictable abrupt transition between two metastable states. Snow avalanches [2], sand avalanches, and woodquakes [3] are some widely known examples of such events in natural systems. Avalanches in interacting networks of strongly nonlinear dynamical nodes are currently considered the main fundamental mechanisms describing biological information processing $[4,5]$.

The avalanches widely explored in physics and technology [6-8] can interfere in the operation of many devices. An example is magnetic avalanches in superconducting resonators [1], implemented in a wide range of systems from quantum bits to devices for research in astrophysics. These avalanches manifest themselves as spontaneous penetrations of magnetic flux in the bulk of the superconductor $[9,10]$. The number of applications based on superconductors increases, and with the important role these devices will play in technology [11], safe operation becomes crucially important.

According to the model based on thermomagnetic instabilities [12], an avalanche is triggered by a thermal

\footnotetext{
*farkhad.aliev@uam.es
}

fluctuation (hot spot), which facilitates more flux motion toward the hot place, with a subsequent heat release. Under quasiequilibrium conditions, the avalanches can also be triggered by microwave pulses $[13,14]$ or ac signals at near resonant frequencies of superconducting cavities [1]. The situation is somewhat different with a broadband microwave (MW) field sweep, with avalanches triggered at different depinning frequencies due complicated local vortex pinning potentials [15].

It is a great challenge to control avalanche processes $[16,17]$. In superconductors, the most common ways to control the fast flux instabilities were through nanomorphology [18] or optimization of the heat transfer through coating a superconducting film with a metal layer [19]. Here, we propose a means of avalanche control using the intrinsic nonlinear MW response of the fundamental units forming the network, superconductor vortices. Such nonlinearity has been recently identified through microwave-stimulated superconductivity due to the presence of vortices [20].

We report a completely unexpected effect: it turns out that the application of microwaves at frequencies close to the vortex depinning frequencies inhibits avalanches. We attribute this effect to a nonlinear dependence of the core size of a dynamically driven vortex on its velocity. This effect allows one to engineer the avalanche behavior, at least to some extent.

\section{EXPERIMENTAL DETAILS}

We study thin films of $\mathrm{Pb}$ with a thicknesses of 60 and $70 \mathrm{~nm}$. These thicknesses are well below the critical value 


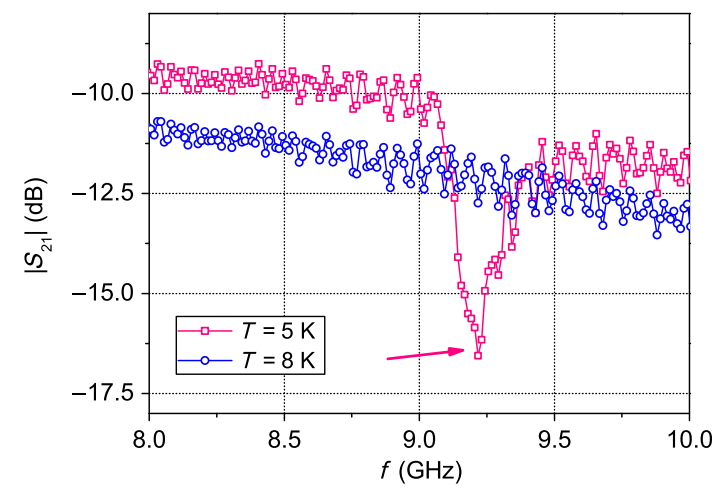

FIG. 1. The arrow points at a depinning frequency, where a large amount of energy is absorbed by the sample in the superconducting state with respect to the normal state.

ensuring type-II superconductivity [21]. In what follows, we present data only from the 70-nm film, since the results observed in both samples are similar. Details in the sample growth have been reported before [20]. The measurements have been done in a variable temperature Janis helium cryostat with a superconducting magnet to apply static magnetic fields. The microwave signal is supplied by a gold coplanar waveguide (CPW). The microwave signal is generated and detected by a network analyzer that carries it to the CPW. More details about this system and the measurement procedure can be found in Ref. [20]. The presence of avalanches is detected as jumps in the normalized transmission parameter $S_{21}$ (we denote this normalized complex quantity as $\left.U=U^{\prime}+i U^{\prime \prime}\right)$, measured by the network analyzer $[1,15]$.

In this work, we focus on the dependence of the microwave power required to trigger avalanches as a function of $T$ and $H$. The microwave frequency has been fixed either to values where the transmission parameter follows a flat tendency both in the superconducting and normal phases, or to specific values in which the superconducting phase exhibits a strong dip in the transmission, at the so-called vortex depinning frequencies $f_{\mathrm{DP}}$ [15] (Fig. 1). The temperature and magnetic field sweeps are extended up to the values destroying the superconductivity (above $T_{c}$ or $H_{c 2}$ ), to use them as a reference.

We also perform computer simulations of the timedependent Ginzburg-Landau (TDGL) equation to propose a model to explain the unusual avalanche behavior with the interplay of vortex displacement and radius under a highfrequency field. This program is described in more detail in Ref. [20]. The results of these simulations are presented with the frequency in units of $f_{0}=96 k_{B} T_{c} / \pi \hbar$ and field (including the high-frequency MW magnetic field) in units of $H_{c 2}$.

\section{RESULTS}

Figure 2(a) sketches the experiment. The superconducting film (blue), grown on top of a substrate (dark gray) is in
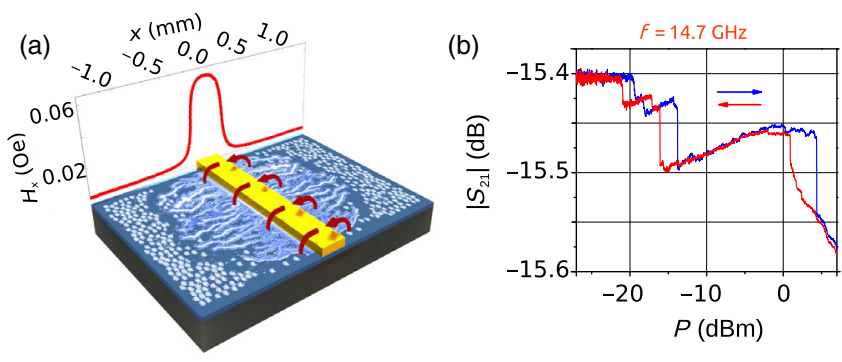

FIG. 2. (a) Sketch of the sample. The graph shows the calculated in-plane magnetic field of the strip line. The red arrows represent the MW field. In light blue is a supposed representation of vortices and avalanches, generated mainly by the strip line. (b) Module of microwave transmission $S_{21}$ vs microwave power, swept from minimum to maximum (blue) or vice versa (red) at a rate of $1 \mathrm{dBm} / \mathrm{s}, T=5 \mathrm{~K}$.

contact with the microwave source (the coplanar waveguide, sketched in yellow). The latter generates a microwave magnetic field (red arrows), incident on the sample, whose parallel-to-the-plane component is shown in the graph in Fig. 2(a). Most of the MW is concentrated close to the strip line, therefore, the high-frequency field exciting the sample is local. This suggests that the avalanches would most probably be generated near the strip line (light blue lines).

Our previous study demonstrated the presence of a set of vortex depinning frequencies in type-II superconducting films [15]. The present experiments aim on the study of these jumps as a function of applied microwave power, for a set of frequencies. The applied field is the remanent one after saturating at 1000 Oe and going back to zero field. In Fig. 2(b), an example of these jumps is presented for a 70-nm-thick $\mathrm{Pb}$ film. Depending on the direction of the power sweep (from the minimum to the maximum value, blue line, or vice versa, red) the jumps appear at different powers. This indicates that the release of an avalanche is influenced by the "history" of the previously released ones. However, at the lowest (or highest) microwave powers, $\left|S_{21}\right|$ is at the same value, indicating that the global superconducting state is equivalent after all these avalanche events. Thus, a "hysteretic" behavior in microwave power evidences the importance of thermal effects in avalanches. Additionally, they show clearly the reproducibility of the power-dependent transmission, so that the jumps in microwave permeability are fully controlled by the applied frequency and MW power. We have not observed substantial changes in the shape of power-dependent permeability on the sweep rate varied between 1 and $10 \mathrm{dBm} / \mathrm{s}$.

Our main findings are presented in Figs. 3(a)-3(d), where we compare a power and temperature sweep of the normalized transmission parameter at $20.2 \mathrm{GHz}$ (a),(c) and $7.5 \mathrm{GHz}$ (b),(d). One notices the previously mentioned [Fig. 2(b)] jumps as steps in the 2D color plot of microwave permeability evident in Fig. 3(a). Moreover, one clearly observes that for temperatures approaching $T_{c}(7.2 \mathrm{~K})$ this 

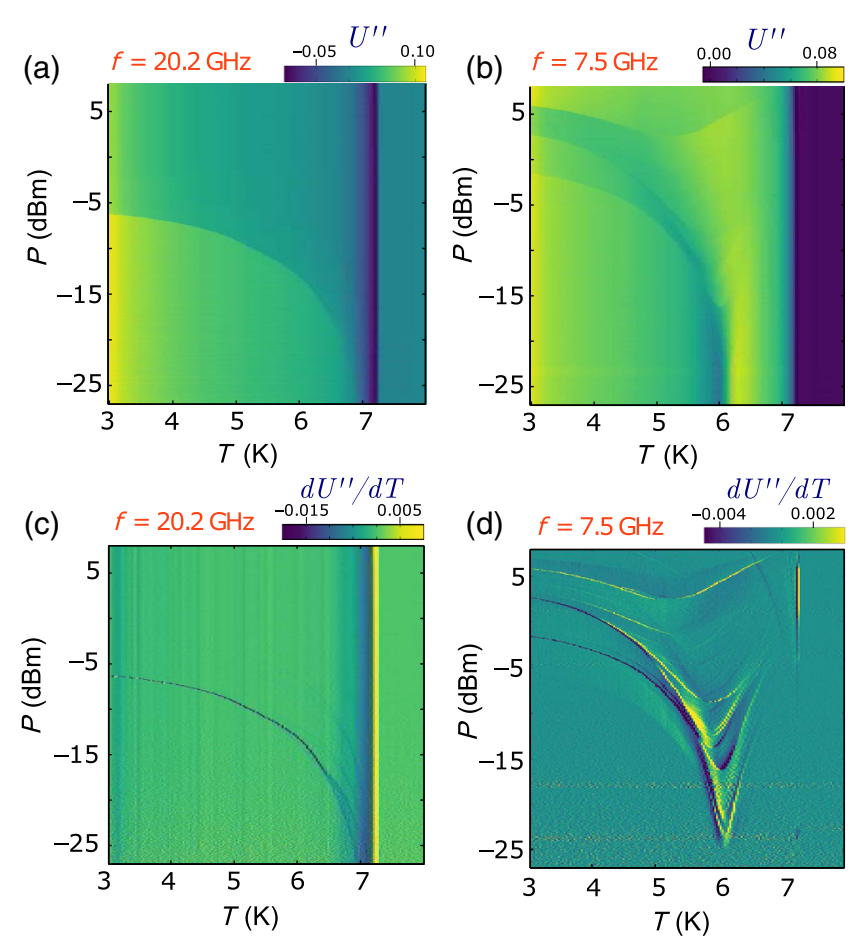

FIG. 3. Microwave stimulated avalanches in a 70-nm film at a freezing field of 10 Oe. Part (a) shows $U^{\prime \prime}$ with expected avalanche behavior vs temperature and power measured at $f=20.2 \mathrm{GHz}$. Part (b) shows $U^{\prime \prime}$ vs microwave power and temperature at different microwave frequency of $f=7.5 \mathrm{GHz}$. Unexpected avalanche behavior vs temperature, close to a depinning frequency is seen. Parts (c) and (d) are derivatives with respect to temperature of the parts (a) and (b). Color bars show $U^{\prime \prime}$ and its derivatives in arbitrary units.

jump requires less MW power to occur. That is in agreement with the expected thermally driven avalanche behavior, since higher temperatures favor the entrance of magnetic flux and enhance the vortex mobility. The corresponding differential plot (derivative with respect to temperature) shown in Fig. 3(c) helps us to observe both the main avalanche (splitting into several approaching $T_{c}$ ). All the avalanches observed outside the vicinities of the depinning frequencies show similar temperature dependences. The critical MW power for triggering an avalanche monotonously decreases while approaching $T_{c}$.

Figure 3(b) shows the same measurements as Fig. 3(a), but close to one of the vortex depinning frequencies, $f_{\mathrm{DP}}=7.5 \mathrm{GHz}$. One clearly observes an unexpected temperature dependence of the critical MW power. Approaching $T_{c}$, within some temperature range (around $6 \mathrm{~K}$ for the lowest powers, and around $5 \mathrm{~K}$ for the strongest ones) the expected tendency of decreasing critical power inverts. The closer one approaches $T_{c}$, the more power is required to trigger the avalanches. We shall further refer to this effect as thermally driven avalanche inhibition (TDAI).

There is no simple relationship between the MW frequency and the presence or absence of the TDAI.

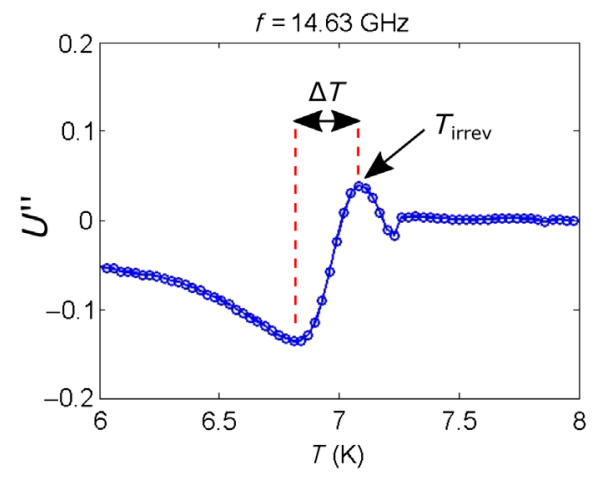

FIG. 4. Temperature dependence of the imaginary contribution to the losses $U^{\prime \prime}$ in the microwave-driven vortex system. The irreversibility temperature is defined as maximum in losses. Also shown is a temperature interval between maximum and minimum in losses which is described with error bars in Fig. 5(e).

A careful examination of the power-temperature and power-field sweeps shows that each time the inversion of the normal tendency to the anomalous one happens, it takes place in a range of temperatures where there is a visible maximum in microwave losses $U^{\prime \prime}$ (see Fig. 4). The peak in MW losses close to the $T_{c}$ is known to originate from the enhanced vortex mobility $[20,22]$.

Figure 5 shows in more detail how the TDAIs vary in the proximity of one of vortex depinning frequencies $f_{\mathrm{DP}}$. At $f<f_{\text {DP }}$ the TDAI effects occur close to $T_{c}$ in the temperature range close to the peak in microwave losses $U^{\prime \prime}$ when the superconducting gap becomes partially filled by quasiparticles and the vortices are mobile. A further increase of the drive frequency near $f=f_{\mathrm{DP}}$ shifts the range where the TDAI takes place. Moreover, as could be observed independently of specific depinning frequencies analyzed in Figs. 3(d) and 5, the higher the applied power is (i.e., less external temperature is needed to create the same vortex mobility), the lower is the temperature at which the corresponding inversion point in the temperature dependence of avalanches occurs. Figure 5(e) shows that the peak in the temperature dependence of $U^{\prime \prime}$ also moves towards lower temperatures as the MW frequency approaches and exceeds the depinning frequency.

At yet higher frequencies $f>f_{\mathrm{DP}}$, the TDAI effects disappear (not shown), as the peak in $U^{\prime \prime}$ vanishes on the lower temperature side of the scale. The scenario repeats again approaching a different vortex depinning frequency. The zero slope point at lower temperatures of the peak in losses shown in Fig. 5(f) represents equal vortex mobility surface as a function of temperature, power, and frequency close to particular vortex depinning frequency.

We have already mentioned that the TDAI may be expected to take place mainly close to the microwave source (the CPW central conductor). A few avalanches could, however, be triggered far from the MW source. Those avalanches should then be subject to a reduced MW power and be thermally stimulated mainly so that their 

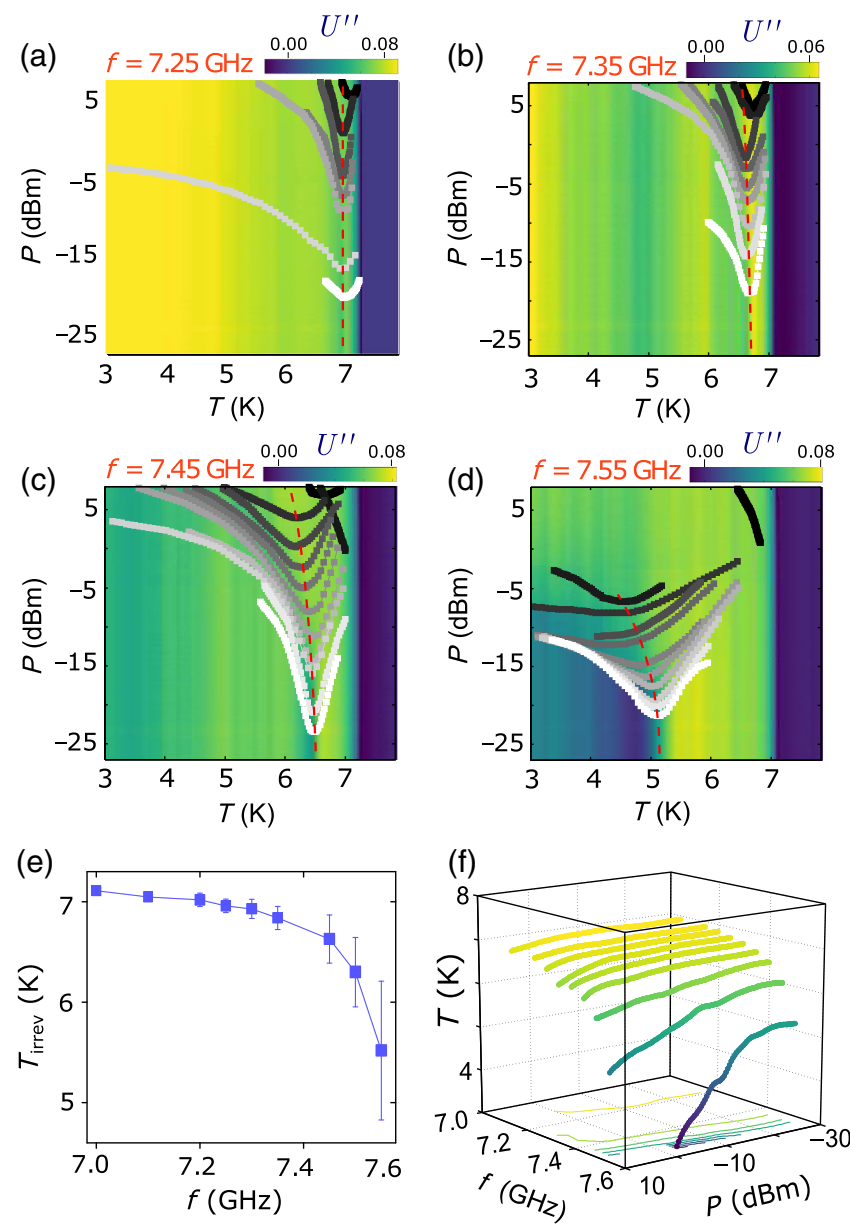

FIG. 5. (a)-(d) Temperature dependence of microwave susceptibility close to one of the depinning frequencies at freezing field. Color bars show $U^{\prime \prime}$ in arbitrary units. Different avalanche branches are indicated in gray tones, extracted from a differential analysis. (e) Temperature of the $U^{\prime \prime}$ peak vs frequency near a depinning frequency, for $P=-20 \mathrm{dBm}$. Error bars represent the temperature difference between the maximum and the base of the peak (see Fig. 4). (f) Dependence of the peak on temperature, power, and frequency.

temperature dependence could not invert approaching the critical temperature or critical field (see Fig. 5 and further below).

Figure 6(a) presents a magnetic field-driven analog of the TDAI effect, but now studied at a fixed temperature $T=$ $5 \mathrm{~K}$ and different MW powers at some higher depinning frequency $f_{\mathrm{DP}}=9.225 \mathrm{GHz}$. Figure 6(c) shows a differential plot $\left(d U^{\prime \prime} / d H\right)$ helping to resolve more clearly the main avalanches marked with dots in (a). In the field range where the peaks in $U^{\prime \prime}$ are observed close to the upper critical field [see Fig. 6(b)], the avalanches show a change in tendency, requiring more power to be triggered at higher fields, where vortex mobility gets enhanced. Compared to temperature sweeps, field sweeps look more complex, with more avalanche branches present, and some of them suffering "jumps" in field as well. This should not be
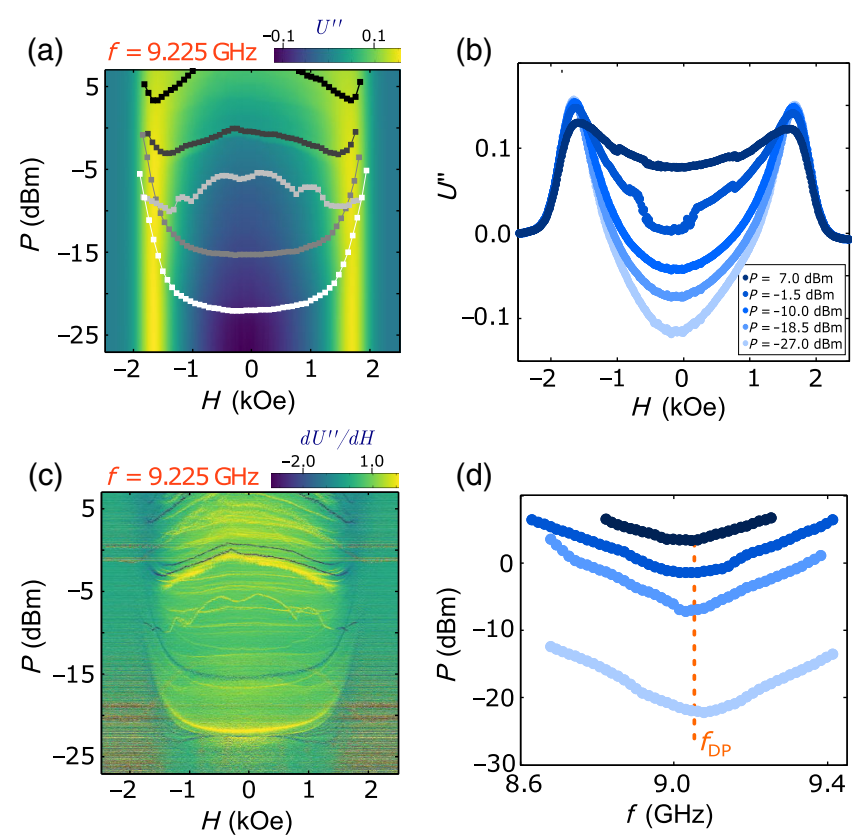

FIG. 6. (a) Microwave losses $U^{\prime \prime}$ as a function of applied field and microwave power measured at $T=0.7 T_{c}(T=5 \mathrm{~K})$ near depinning frequency $f_{\mathrm{DP}}=9.225 \mathrm{GHz}$. (b) Cross sections of (a) at different powers, highlighting the peaks in losses near the critical field. (c) Differential plot $\left(d U^{\prime \prime} / d H\right)$ of (a). It allows a more clear detection of avalanches, marked with dots in (a). (d) Different frequency-dependent avalanches close to the depinning frequency of (a).

surprising, as changing the applied field changes the number of vortices present in the sample and enhances nonequilibrium, which stimulates the avalanche process.

Figure 6(d) shows how critical power needed to stimulate avalanches varies as a function of frequency close to some specific $f_{\mathrm{DP}}=9.225 \mathrm{GHz}$. At a fixed temperature $\left(T / T_{c}=0.7\right)$ and some small magnetic field $(H \simeq 100 \mathrm{Oe})$ the MW power needed to trigger avalanches reduces nearly symmetrically with frequency relative to the depinning frequency.

\section{MODEL AND DISCUSSION}

The experimentally observed unforeseen thermally induced enhanced stability of the vortex avalanches to the external microwave excitation could be understood as a consequence of a counterintuitive dependence of the vortex core size on the MW power and frequency.

Larkin and Ovchinnikov (LO) were the first to point out a strongly nonlinear dependence on the vortex core size on the vortex velocity [23]. They predicted a reduction of the vortex core size as the vortex velocity approaches some critical value. As a consequence of the LO effect, the size of the core of a periodically driven vortex changes in time. In order to understand the experimental results we consider for simplicity three qualitatively different regimes. In the regimes (a) with low or (b) with high MW power 

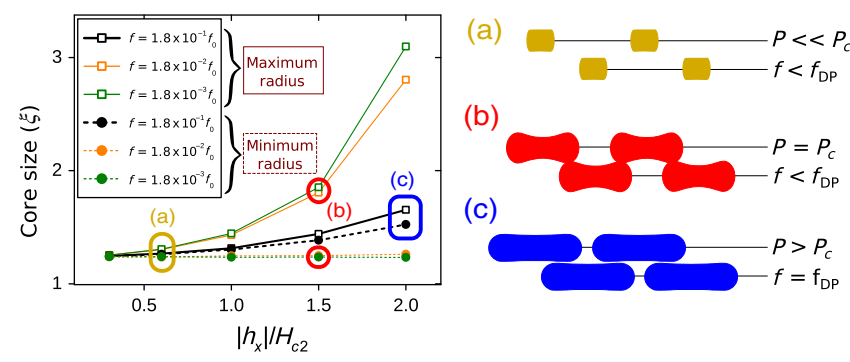

(b)

(c)

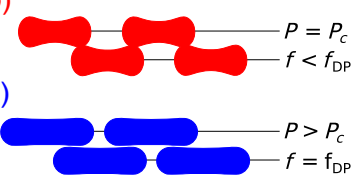

FIG. 7. Simulations of the change in vortex core size during an oscillation period for different frequencies and amplitudes of rf field. The values of radius are taken at $|\Psi|=0.5$. The sketch at the right illustrates the overlap of the regions where vortices move under microwave fields of different powers. As vortices move, their radius change. This could allow heated zones to overlap to produce avalanches. The situations (a), (b), and (c) correspond to those indicated in the graph with the same letters and colors.

(microwave magnetic field) but outside the vortex depinning regime, the LO effect manifests itself only in the regions of maximal velocity (i.e., close to displacement minima). On the other hand, in the regime (c), which is close to a depinning frequency, the vortices become more mobile. As a result, the LO reduction of vortex core manifests itself during the whole displacement cycle.

To elucidate the physics of the thermally inhibited avalanches in the driven vortex system, following the approach [20], we have simulated the relative changes in the vortex core size with both drive frequency and microwave power. Figure 7 explains the model describing a transition from thermally activated to thermally suppressed vortex avalanches approaching a depinning frequency. Based on the simulation results, we conclude that for the driving frequencies below the depinning frequency and low MW power $P$ local "normal" regions created by the driven vortex do not overlap and are not sufficient to trigger the avalanche process [case (a), marked in yellow]. Note that the depinning frequency $f_{\text {DP }}$ plays the role of a critical value above which the core size shrinks under the MW drive [20]. Increasing the MW power, but still below the depinning frequency, enhances the absolute changes in the vortex core size (Fig. 7), inducing the overlap between different extended core areas and triggering the avalanche close to some critical power $P=P_{c}$ [case (b) in simulations marked in red]. Evidently, such a process is thermally activated, as long as the vortex diameter strongly increases approaching critical temperature $T_{c}$.

However, close to depinning frequencies and in the temperature region where vortices become depinned (i.e., at temperatures about $T_{\mathrm{DP}}$ ), the $\mathrm{LO}$ effect reduces the vortex core ending up in only a small variation in the vortex core size during periodically driven motion [case (c), Fig. 7]. This reduces the probability of the overlap between normal regions and, therefore, the avalanche should be triggered at yet higher applied MW powers for the fixed temperature, exactly as we observe experimentally.
The proposed scenario suggests only the basic mechanism behind the TDAI effect. Specific forms of the normal areas occupied by the MW-driven vortex should depend on the pinning details and thermal energy relaxation rate outside the vortex core. However, once the percolation on the local level between the "hot spots" created by the driven vortices is established, the avalanche could be triggered with high probability. Superconducting vortex avalanches are usually described by a thermomagnetic instability. In this model, the avalanches develop as a result of the heat released due to flux motion leading to enhancement of the local vortex mobility and, correspondingly, further heat release. This positive feedback balanced by heat dissipation towards the substrate results in ultrafast dendritic flux redistributions. Our work shows that (contrary to expectations) close to depinning frequencies the thermal bath energy may inhibit the avalanche processes due to a nonlinear dependence of the vortex core on the microwave field.

\section{CONCLUSIONS}

In conclusion, we have systematically studied the microwave-driven superconducting vortex avalanches at different frequencies and intensities of MW radiation. Both the cases of weak and strong overlap of the vortex cores are investigated. The main finding is that magnetic avalanches in microwave-driven superconducting vortex systems close to depinning frequencies can become more robust to external stimulus. This is in contrast with the usually observed thermal weakening of the avalanche process. A simple model which considers shrinking in size of the microwave-driven vortex qualitatively explains the main observations. The observed effects point towards potential robustness of superconducting devices to stimulated avalanche processes when external drive frequencies are tuned to vortex depinning frequencies.

\section{ACKNOWLEDGMENTS}

The authors gratefully acknowledge A. Awad and A. Silhanek for experimental help on the initial stages and $\mathrm{K}$. Ilin for discussions. This work has been supported in part by Spanish MINECO (MAT2015-66000-P), and Comunidad de Madrid (NANOFRONTMAG-CM S2013/ MIT-2850) and NANO-SC COST-Action MP-1201. V. V. M. acknowledges the Methusalem Funding of the Flemish Government. A. L. thanks Comunidad de Madrid for the postdoctoral contract through Consejería de Educación, Juventud y Deporte de la Comunidad de Madrid y Fondo Social Europeo.

[1] G. Ghigo, F. Laviano, L. Gozzelino, R. Gerbaldo, E. Mezzetti, E. Monticone, and C. Portesi, Evidence of rf-driven dendritic vortex avalanches in $\mathrm{MgB}_{2}$ microwave resonators, J. Appl. Phys. 102, 113901 (2007). 
[2] J. Qiu, Avalanche hotspot revealed, Nature (London) 509, 142 (2014).

[3] T. Mkinen, A. Miksic, M. Ovaska, and Mikko J. Alava, Avalanches in Wood Compression, Phys. Rev. Lett. 115, 055501 (2015).

[4] R. Stoop and F. Gomez, Auditory Power-Law Activation Avalanches Exhibit a Fundamental Computational Ground State, Phys. Rev. Lett. 117, 038102 (2016).

[5] J. M. Beggs and D. Plenz, Neuronal avalanches in neocortical circuits, J. Neurosci. 23, 11167 (2003).

[6] M. Nada, M. Nakamura, and H. Matsuzaki, 25-Gbit/s burstmode optical receiver using high-speed avalanche photodiode for 100-Gbit/s optical packet switching, Opt. Express 22, 443 (2014).

[7] G. Bulgarini, M. E. Reimer, M. Hocevar, E. P. A. M. Bakkers, L. P. Kouwenhoven, and V. Zwiller, Avalanche amplification of a single exciton in a semiconductor nanowire, Nat. Photonics 6, 455 (2012).

[8] O. Jukimenko, C. M. Dion, M. Marklund, and V. Bychkov, Multidimensional Instability and Dynamics of Spin Avalanches in Crystals of Nanomagnets, Phys. Rev. Lett. 113, 217206 (2014).

[9] S. Field, J. Witt, F. Nori, and X. Ling, Superconducting Vortex Avalanches, Phys. Rev. Lett. 74, 1206 (1995).

[10] T. H. Johansen, M. Baziljevich, D. V. Shantsev, P. E. Goa, Y. M. Galperin, W. N. Kang, H. J. Kim, E. M. Choi, M.-S. Kim, and S. I. Lee, Dendritic magnetic instability in superconducting $\mathrm{MgB}_{2}$ films, Europhys. Lett. 59, 599 (2002).

[11] Y. Wang, Fundamental Elements of Applied Superconductivity in Electrical Engineering (John Wiley \& Sons, Inc., Singapore, 2013).

[12] A. L. Rakhmanov, D. V. Shantsev, Y. M. Galperin, and T. H. Johansen, Finger patterns produced by thermomagnetic instability in superconductors, Phys. Rev. B 70, 224502 (2004).

[13] A. V. Bobyl, D. V. Shantsev, T. H. Johansen, W. N. Kang, H. J. Kim, E. M. Choi, and S. I. Lee, Current-induced dendritic magnetic instability in superconducting $\mathrm{MgB}_{2}$ films, Appl. Phys. Lett. 80, 4588 (2002).
[14] P. J. Cuadra-Solís, J. M. Hernández, A. García-Santiago, J. Tejada, J. Vanacken, and V. V. Moshchalkov, Avalanchelike vortex penetration driven by pulsed microwave fields in an epitaxial LaSrCuO thin film, J. Appl. Phys. 114, 233902 (2013).

[15] A. A. Awad, F. G. Aliev, G. W. Ataklti, A. Silhanek, V. V. Moshchalkov, Y. M. Galperin, and V. Vinokur, Flux avalanches triggered by microwave depinning of magnetic vortices in $\mathrm{Pb}$ superconducting films, Phys. Rev. B 84, 224511 (2011).

[16] Y. C. Zohar, S. Yochelis, K. A. Dahmen, G. Jung, and Y. Paltiel, Controlling avalanche criticality in 2D nano arrays, Sci. Rep. 3, 1845 (2013).

[17] G. Curcio, E. Mazzucchi, G. Della Marca, C. Vollono, and P. M. Rossini, Electromagnetic fields and EEG spiking rate in patients with focal epilepsy, Clin. Neurophysiol. 126, 659 (2015).

[18] V. V. Yurchenko, K. Ilin, J. M. Meckbach, M. Siegel, A. J. Qviller, Y.M. Galperin, and T.H. Johansen, Thermo-magnetic stability of superconducting films controlled by nano-morphology, Appl. Phys. Lett. 102, 252601 (2013).

[19] E. M. Choi, V. V. Yurchenko, T. H. Johansen, H. S. Lee, J. Y. Lee, W. N. Kang, and S. I. Lee, Suppression of dendritic flux jumps in $\mathrm{MgB}_{2}$ films coated with a gold rim, Supercond. Sci. Technol. 22, 015011 (2009).

[20] A. Lara, F. G. Aliev, A. V. Silhanek, and V. V. Moshchalkov, Microwave-stimulated superconductivity due to presence of vortices, Sci. Rep. 5, 9187 (2015).

[21] G. J. Dolan and J. Silcox, Critical Thicknesses in Superconducting Thin Films, Phys. Rev. Lett. 30, 603 (1973).

[22] M. W. Coffey and J. R. Clem, Theory of rf magnetic permeability of isotropic type-II superconductors in a parallel field, Phys. Rev. B 45, 9872 (1992).

[23] A. I. Larkin and Y. N. Ovchinnikov, Nonlinear conductivity of superconductors in the mixed state, Sov. Phys. JETP 41, 960 (1975). 